\title{
Análisis teórico de una chimenea solar con tres canales de flujo de aire
}

\section{Theoretical analysis of a solar chimney with three air flow channels}

\author{
TLATELPA-BECERRO, Ángel ${ }^{1,2} \dagger^{*}$, RICO-MARTÍNEZ, Ramiro ${ }^{3}$, URQUIZA-BELTRÁN, Gustavo ${ }^{4}$ y \\ REYNOSO-JARDÓN, Elva Lilia ${ }^{5}$
}

\author{
${ }^{I}$ Escuela de Estudios Superiores de Yecapixtla, UAEM. \\ ${ }^{2}$ Universidad Politécnica de Atlautla, UPA. \\ ${ }^{3}$ Centro de Investigación en Ingeniería y Ciencias Aplicadas, UAEM. \\ ${ }^{4}$ Instituto Tecnológico de Celaya, TECNM. \\ ${ }^{5}$ Universidad Autónoma de Ciudad Juárez, UACJ.
}

\author{
ID $1^{\mathrm{er}}$ Autor: Angel, Tlatelpa-Becerro / ORC ID: 0000-0002-4891-4405, CVU CONACYT ID: 264442 \\ ID $2^{\text {do }}$ Coautor: Ramiro, Rico-Martínez/ ORC ID: 0000-0001-7033-7432, CVU CONACYT ID: 12424 \\ ID $3^{\text {do }}$ Coautor: Gustavo, Urquiza-Beltrán / CVU CONACYT ID: 5222
}

ID $4^{\text {to }}$ Coautor: Elva Lilia, Reynoso-Jardón / ORC ID: 0000-0002-0729-2822, CVU CONACYT ID: 264446

DOI: $10.35429 / J T O .2019 .12 .3 .13 .19$

Recibido 26 de Octubre, 2019, Aceptado, 02 de Diciembre, 2019

\begin{abstract}
Resumen
Se propone una configuración de chimenea solar, que consiste en tres canales de flujo de aire divididos por dos placas metálicas, colocadas al centro de la chimenea entre dos cubiertas acrílicas, conduciendo al flujo de aire simétrico, como una alternativa para el diseño de sistemas de ventilación natural en edificios para zonas climáticas tropicales y subtropicales. Las dimensiones de chimenea solar son $2.0 \mathrm{~m}$ de altura, $1.0 \mathrm{~m}$ de ancho, y el hueco entre canales de $0.30 \mathrm{~m}$. Estas dimensiones son apropiadas para el diseño de sistemas de ventilación natural para viviendas residenciales en el centro de México. Una simulación numérica aplicando balances globales de masa y energía en estado estacionario fue utilizada para evaluar la eficiencia de la configuración propuesta. Los perfiles de temperaturas, calculados para un día típico caluroso en una región tropical, revelan que la configuración es más eficiente que la configuración de un solo canal, alcanzando valores de eficiencia térmica cercanos al $75 \%$. La configuración de la chimenea solar propuesta puede ser utilizada con mejores resultados que el diseño tradicional de un solo canal como alternativa de ventilación natural para viviendas residenciales sin un incremento sensible en la inversión de la residencia.
\end{abstract}

Chimenea solar, Ventilación natural, Energía solar

\begin{abstract}
A solar chimney configuration consisting of three air flow channels divided by two metallic plate, placed in the center of the chimney between two acrylic covers, leading to symmetric air flow, is proposed as an alternative for the design of natural ventilation systems for buildings in tropical and subtropical climatic zones The solar chimney dimensions are $2.0 \mathrm{~m}$ height, $1.0 \mathrm{~m}$ width, and gap between channels of $0.30 \mathrm{~m}$. These dimensions are appropriate for the design of ventilation systems for residential buildings in central México. A Numerical simulation using the global mass and energy balances in steady state was utilized to evaluate the efficacy of the proposed configuration. The temperature profiles, calculated for a typical hot day in a tropical region, reveal that the configuration is more efficient than the single channel chimney, achieving thermal efficiency values near $75 \%$. This solar chimney configuration can be used with better results than the traditional design as an alternative for natural ventilation systems in residential buildings without a significant increase in the cost of the residence investment.
\end{abstract}

Solar chimney, Natural ventilation, Solar energy

Citación: TLATELPA-BECERRO, Ángel, RICO-MARTÍNEZ, Ramiro, URQUIZA-BELTRÁN, Gustavo y REYNOSOJARDÓN, Elva Lilia. Análisis teórico de una chimenea solar con tres canales de flujo de aire. Revista de Operaciones Tecnológicas. 2019 3-12: 13-19

\footnotetext{
* Correspondencia del Autor (Correo electrónico: angel.tlatelpa@uaem.mx)

$\dagger$ Investigador contribuyendo como primer autor.
} 


\section{Introducción}

Hoy en día, la ventilación natural se considera un de los requerimientos en el diseño de edificios para un consumo eficiente de energía. La ventilación natural es un sistema de acondicionamiento pasivo utilizado para la comodidad y el bienestar humano. Este sistema se caracteriza por ser independiente del suministro de energía convencional. De esta manera, su contribución para reducir las emisiones de gases de efecto invernadero está ganando importancia. El desafío es buscar diseños y alternativas para reducir el consumo de energía convencional. El uso de fuentes de energía renovable, por ejemplo, como la energía solar a través de diferentes sistemas de ventilación pasiva para los edificios es una de las vías para enfrentar este desafío (Awbi, 2003).

Algunos estudios han sido explorados durante los últimos 25 años, entre ellos (Bansal et al., 1993) han analizado una habitación acoplada a un colector solar con propósitos de ventilación, logrando una buena variación de condiciones de enfriamiento para la habitación. (Ong, 2003 y Hirunlab et al., 1999) propusieron el uso de la metodología de balances globales para análisis similares. (Lee and Strand, 2009) determinaron el impacto energético de una chimenea solar a través de simulación numérica con datos para condiciones climatológicas y flujos másicos diferentes. Desde estos estudios pioneros, se han utilizado varios modelos y software de simulación para el análisis de los sistemas de ventilación natural. Por ejemplo, (Imran et al., 2015) estudiaron el efecto de la incidencia de la radiación solar directa sobre una chimenea solar inclinada; (Ghalamchi et al., 2015) uso la simulación en busca de una configuración óptima. Otros autores han abordado la geometría y la configuración para mejorar la eficiencia de los sistemas (Sandeep et al., 2014; Gholamalizadeh and Kim, 2016; Zhongting et al., 2018, Saifi et al., 2012; Karima et al., 2012). Sin embargo, todavía faltan directrices claras para el diseño eficiente y la selección de chimeneas solares.

En esta contribución, se desarrolla un modelo matemático para una chimenea solar con tres canales de flujo de aire en flujo unidimensional. La eficiencia térmica del sistema se examina bajo diferentes condiciones de irradiancia solar, temperatura y velocidad del aire.
Los datos utilizados corresponden para un horario de 10:00 a 17:00 hrs, estos fueron registrados para un día despejado del 3 de junio de 2010 en Tlaquiltenango Morelos, México. Con latitud y longitud de $18.370001^{\circ} \mathrm{LN}$ y 99.029999 LW, respectivamente.

\section{Modelo Físico}

La Figura 1 muestra un diagrama esquemático de la chimenea solar, ubicada en la parte más soleada del edificio. En la figura 2 se muestra la sección transversal de la chimenea solar. Los elementos principales de la configuración son dos placas metálicas ( 1 y 2 ) colocadas en el centro entre dos cubiertas acrílicas que forman simétricamente tres canales de flujo de aire $\left(\mathrm{C}_{1}\right.$, $\mathrm{C}_{2}$ y $\mathrm{C}_{3}$ ). Las placas metálicas están pintadas en negro mate, para la absorción de radiación solar durante el día. La placa debe colocarse de tal manera que reciba radiación solar en una de sus caras (cara este) hasta el mediodía; después del mediodía se recibe radiación solar en la otra cara (cara oeste), para aprovechar la máxima captación de radiación solar durante el día. Las dimensiones de la chimenea solar son: $d_{1}=$ $d_{2}=d_{3}=0.30 \mathrm{~m}$ espacio entre cada canal de aire, $L=2.0 \mathrm{~m}$ alto de la chimenea, ver Fig. 3 .

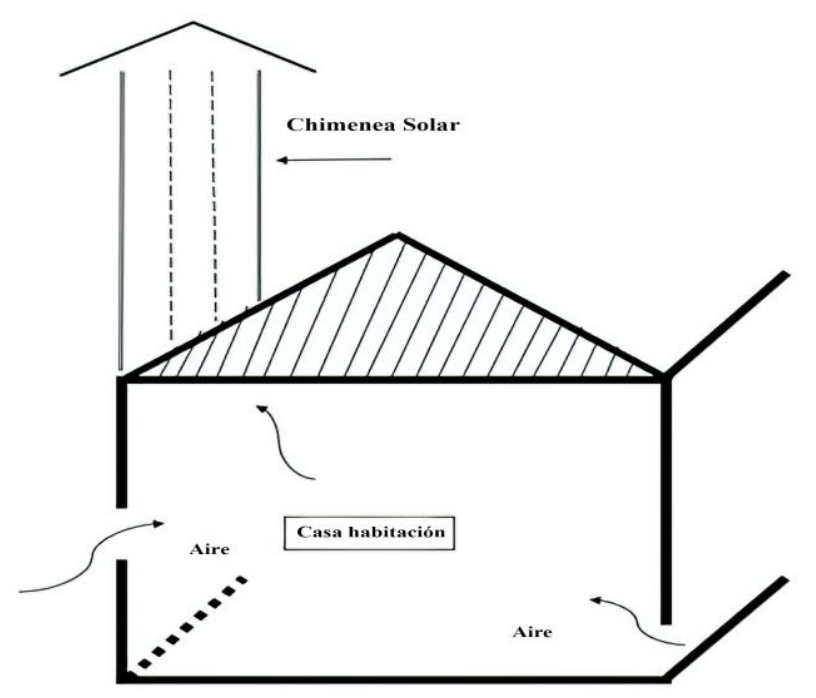

Figura 1 Diagrama esquemático de la chimenea solar Fuente: Elaboración Propia

Las cubiertas acrílicas se utilizan para disminuir las pérdidas por convección e irradiación. Se consideran transparentes, y de 4 $\mathrm{mm}$ de espesor. La cubierta acrílica absorbe, transmite y refleja la radiación solar incidente. Las placas metálicas absorben la mayor parte de la energía solar incidente, donde la energía restante sufre otros procesos de transferencia de calor: radiación, convección y conducción, como se muestra en la Figura 3.

TLATELPA-BECERRO, Ángel, RICO-MARTÍNEZ, Ramiro, URQUIZA-BELTRÁN, Gustavo y REYNOSO-JARDÓN, Elva Lilia. Análisis teórico de una chimenea solar con tres canales de flujo de aire. Revista de Operaciones Tecnológicas. 2019 


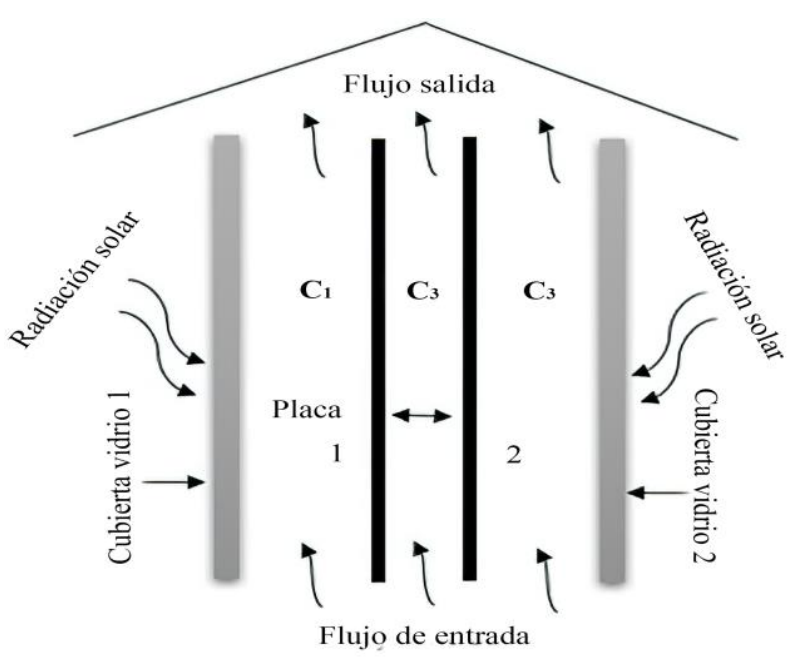

Figura 2 Sección transversal de la chimenea solar Fuente: Elaboración Propia

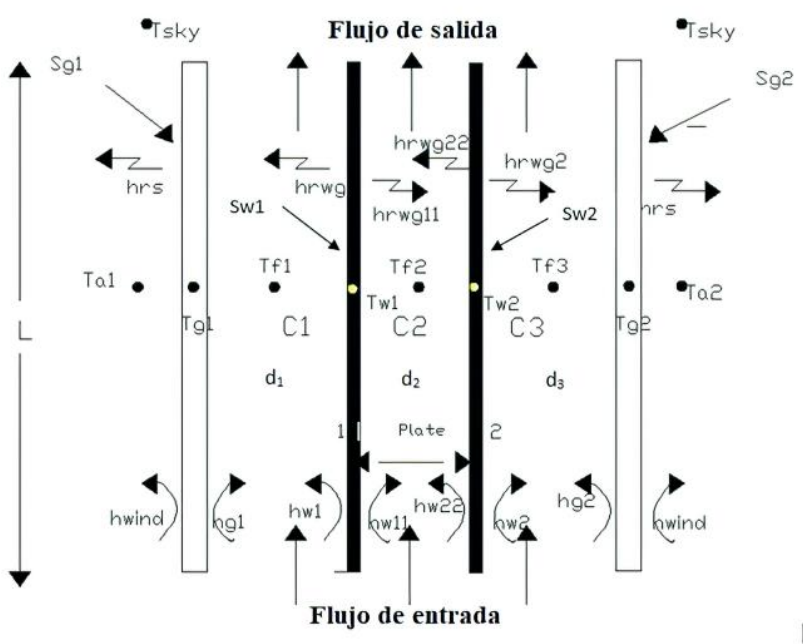

Figura 3 Modelo Físico

Fuente: Elaboración Propia

En la Figura 3, $S_{g 1}$ y $S_{g 2}$ representan la irradiancia solar.

\section{Modelo Matemático}

La metodología de balances globales se aplica para estudiar esta configuración, y está descrita por (Ong, 2003). Se consideran las siguientes suposiciones:

- $\quad$ Estado estable. No hay variaciones de tiempo.

- Todos los procesos de transferencia de energía a través de la cubierta acrílica, la placa absorbente y el canal de aire son unidimensionales.

- Las placas metálicas y las cubiertas acrílicas son paralelas y orientadas verticalmente.

- $\quad$ La temperatura de entrada del aire en el canal de flujo es igual a la temperatura ambiente.
- $\quad$ Las pérdidas por fricción en el colector son despreciables debido a la muy baja velocidad de flujo.

Todas las propiedades termofísicas se consideran constantes y se evalúan a una temperatura promedio.

La Figura 4 muestra una red térmica análoga a los circuitos resistivos para los componentes de la chimenea solar.

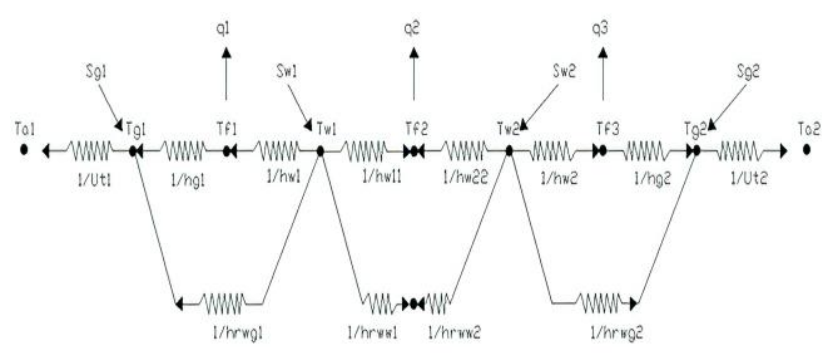

Figura 4 Red térmica de la chimenea solar.

Fuente: Elaboración Propia

Teniendo en cuenta las suposiciones anteriores y la red térmica que se muestra en la Figura 4, y siguiendo la primera ley de la Termodinámica, la ecuación de conservación de energía se puede escribir como (Yunus y Michael, 2015), ecuación (1):

$\dot{E}_{\text {in }}+\dot{E}_{g}-\dot{E}_{\text {out }}=\dot{E}_{\text {st }}$

Las ecuaciones de balance de energía se expresan directamente en forma de matriz dado el sistema de 7 ecuaciones algebraicas, y se expresan en la ecuación (2):

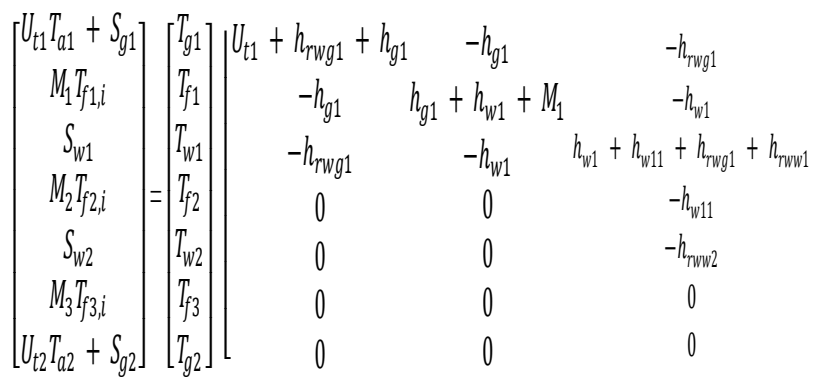
$\left.\begin{array}{cccc}0 & 0 & 0 & 0 \\ 0 & 0 & 0 & 0 \\ -h_{w 11} & -h_{\text {rww1 }} & 0 & 0 \\ h_{w 11}+h_{w 22}+M_{2} & -h_{w 22} & 0 & 0 \\ -h_{w 22} & h_{w 2}+h_{w 22}+h_{\text {rwg2 }}+h_{\text {rww2 }} & -h_{w 2} & 0 \\ 0 & -h_{w 2}+h_{g 2}+M_{3} & -h_{g 2} \\ 0 & -h_{\text {hwg2 }} & -h_{g 2} & U_{t 2}+h_{\text {rwg2 }}+h_{g 2}\end{array}\right]$ (2)

TLATELPA-BECERRO, Ángel, RICO-MARTÍNEZ, Ramiro, URQUIZA-BELTRÁN, Gustavo y REYNOSO-JARDÓN, Elva Lilia. Análisis teórico de una chimenea solar con tres canales de flujo de aire. Revista de Operaciones Tecnológicas. 2019 
Donde la columna $T_{g 1}, T_{g 2}$, $T_{w 1}, T_{w 2}, T_{f 1}, T_{f 2}, T_{f 3}$, es el vector de temperatura desconocido. Incluye las temperaturas de la cubierta acrílica, las temperaturas de las placas metálicas y las temperaturas del aire en cada uno de los tres canales, respectivamente. El vector de temperatura se calcula resolviendo el sistema matricial.

La irradiación solar absorbida por la cubierta, en ambos lados, viene dada por la ecuación (3):

$S_{g}=\alpha_{1} G$

La irradiación solar transmitida que cubre la placa metálica, en ambos lados, se define mediante la ecuación (4):

$S_{w}=\alpha \tau G$

El coeficiente de pérdida para la parte superior de la chimenea y el coeficiente de transferencia de calor del viento se evalúan mediante las ecuaciones (5) y (6):

$U_{t}=h_{\text {wind }}+h_{r s}$

$h_{\text {wind }}=5.7+3.8 \mathrm{~V}$

El coeficiente de transferencia de calor por radiación desde la cubierta acrílica a la bóveda celeste (Duffie y Beckman, 1991) se evalúa mediante la ecuación (7), donde $T_{s}$ es la temperatura de la bóveda celeste, que se define mediante la ecuación (8).

$h_{r s}=\frac{\sigma \varepsilon_{1}\left(T_{g}+T_{s}\right)\left(T_{g}^{2}+T_{S}^{2}\right)\left(T_{g}-T_{S}\right)}{\left(T_{g}+T_{a}\right)}$

$T_{\text {sky }}=0.0552 T_{a}^{3 / 2}$

El coeficiente de transferencia de calor por radiación entre la placa absorbente y la cubierta acrílica también se evalúa según (Duffie y Beckman, 1991), como se indica a continuación, la ecuación (9):

$h_{r w g}=\frac{\sigma\left(T_{g}^{2}+T_{w}^{2}\right)\left(T_{g}+T_{w}\right)}{\left(\frac{1}{\varepsilon_{g}}+\frac{1}{\varepsilon_{w}}-1\right)}$
Los coeficientes de transferencia de calor por convección para la convección natural se determinan después de aplicar la correlación de Nusselt, para flujo laminar, ecuación (10), para flujo turbulento, ecuación (11), con el número de Rayleigh $\quad R_{a} \leq 10^{9} \quad$ and $R_{a} \geq 10^{9}$, respectivamente. (Incropera and Witt, 2002), ecuación. (10) and (11).

$$
\begin{aligned}
& N u=0.68+\left(0.67 R a^{1 / 4}\right) /\left[1+(0.492 / P r)^{9 / 16}\right]^{4 / 9} \\
& N u=\left\{0.825+\left(0.67 R a^{1 / 6}\right) /\left[1+(0.492 / P r)^{9 / 16}\right]^{8 / 27}\right\}^{2}
\end{aligned}
$$

Donde los números de Rayleigh y Prandtl están dados por las ecuaciones (12) y (13):

$$
\begin{aligned}
& R a=\frac{g \beta \Delta T L^{3}}{v \alpha} \\
& P r=\frac{c_{f} \mu_{f}}{k_{f}}
\end{aligned}
$$

Las siguientes correlaciones matemáticas fueron obtenidas para las propiedades termofísicas y que fueron calculadas usando la temperatura promedio del fluido (Incropera y Witt, 2002):

Viscosidad dinámica, ecuación. (14):

$\mu_{f}=\left[2.2078\left[T_{f}^{0.7756}\right]\right] \times 10^{-7}$

Densidad, ecuación. (15):

$\rho_{f}=-0.0021\left[T_{f}\right]+1.7511$

Conductividad térmica, ecuación. (16):

$k_{f}=\left[0.1723\left[T_{f}^{0.8812}\right]\right] \times 10^{-3}$

Calor específico, ecuación. (17):

$C_{f}=\left[0.9125\left[T_{f}^{0.0174}\right]\right] \times 10^{3}$

El coeficiente de expansión volumétrico se define mediante la ecuación (18) y se evalúa a una temperatura media utilizando la ecuación (19) y $M$ mediante la ecuación (20).

$$
\begin{aligned}
& \beta_{f}=\frac{1}{T_{m}} \\
& T_{m}=\frac{T_{g}+T_{w}}{2} \\
& M=\frac{\dot{m} C_{f}}{W \Delta x y}
\end{aligned}
$$


El flujo másico se evalúa usando la fórmula sugerida por (Bansal et al., 2005), ecuación. (21).

$\dot{m}=C_{d} \frac{\rho_{a i r, o} A_{0}}{\sqrt{1+A_{r}^{2}}} \sqrt{\frac{2 g L\left(T_{f, i}-T_{r}\right)}{T_{r}}}$

Donde la relación de aspecto se define mediante la ecuación (22):

$A_{r}=\frac{A_{o}}{A_{i}}$

La eficiencia térmica se determina utilizando la siguiente ecuación (23):

$\eta=\frac{\dot{m} c_{f}\left(T_{f, 0}-T_{f, i}\right)}{W \cdot L \cdot G} x 100$

Este modelo matemático está dispuesto en forma matricial y codificado en FORTRAN. Se utiliza un algoritmo iterativo para predecir los valores de la cubierta, $T_{g}$, placa absorbente, $T_{w}$, las temperaturas del fluido del aire, $T_{f}$ y el caudal másico de aire, $\dot{m}$. Los valores de las propiedades ópticas de los materiales se muestran en la Tabla 1.

\begin{tabular}{|l|c|c|c|}
\hline \multicolumn{1}{|c|}{ Material } & \multicolumn{1}{c|}{ Emisividad } & \multicolumn{1}{c|}{ Absortividad } & Transmisividad \\
\hline $\begin{array}{l}\text { Cubierta } \\
\text { acrílica }\end{array}$ & 0.83 & 0.06 & 0.84 \\
\hline $\begin{array}{l}\text { Cubierta } \\
\text { acrílica }\end{array}$ & 0.83 & 0.06 & 0.84 \\
\hline $\begin{array}{l}\text { Pintura } \\
\text { negra }\end{array}$ & 0.91 & 0.95 & 0 \\
\hline $\begin{array}{l}\text { Pintura } \\
\text { negra }\end{array}$ & 0.91 & 0.95 & 0 \\
\hline
\end{tabular}

Tabla 1 Propiedades óticas de los materiales Fuente: Modest, 2003; Mills, 1999

\section{Resultados y discusiones}

Los datos meteorológicos utilizados para este estudio se obtienen en la Estación Meteorológica ubicada en la ciudad de Tlaquiltenango, estado de Morelos, México, a $18^{\circ} 37$ " msnm. Los datos fueron registrados para un día claro correspondiente al 3 de junio de 2010.

La Figura 5 muestra la radiación solar (global, difusa y directa) y la velocidad del aire, mientras que en la Figura 6 se puede observar la irradiación solar máxima para ambas orientaciones, este y oeste, así como la temperatura del aire. Estos datos, se registran durante las 24 horas del día.

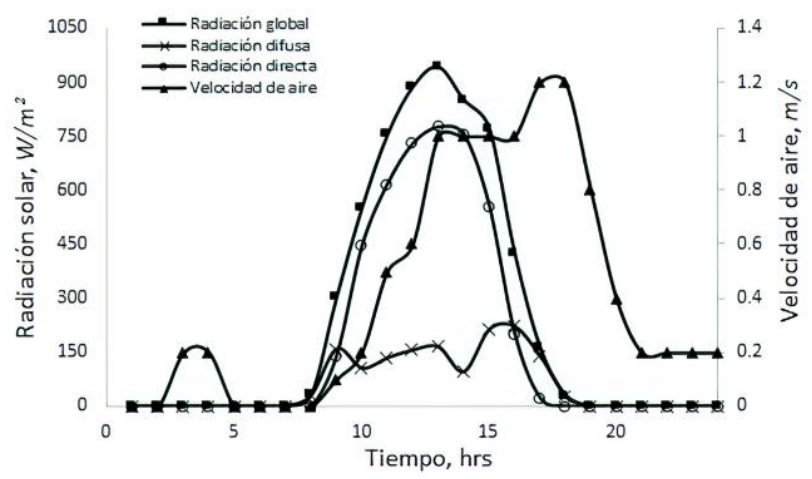

Figura 5 Radiación solar y velocidad del aire para 24 hrs del día

Fuente: Elaboración Propia

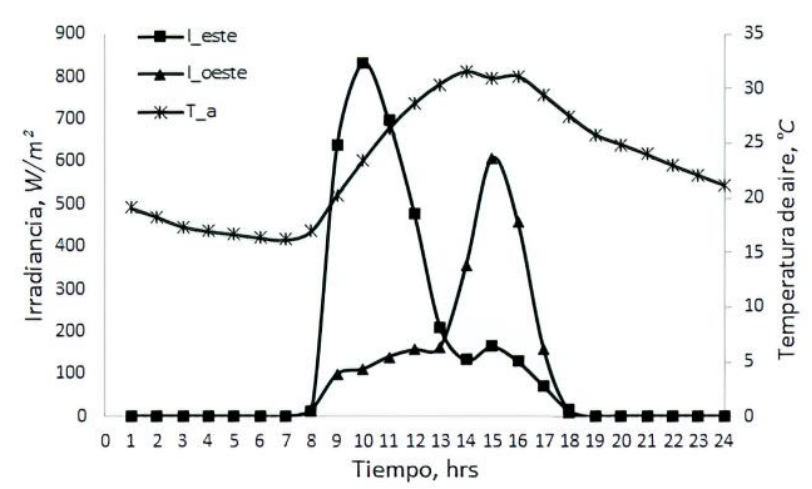

Figura 6 Irradiancia solar y temperatura del aire para 24 hrs del día

Fuente: Elaboración Propia

Datos registrados de irradiación solar, temperatura y velocidad del aire de 10:00 a 17:00 hrs. del día se utilizan para ejecutar las simulaciones y predecir los flujos másicos y las eficiencias.

Las curvas de la temperatura de las cubiertas acrílicas, la temperatura de las placas metálicas y la temperatura del aire se representan en función de la irradiancia solar, como se muestra en la Figura 7.

Todas las temperaturas promedio aumentan con respecto a la irradiancia solar observada.

Las temperaturas medias más altas obtenidas corresponden a las placas metálicas, Figura 7, b). Además, en la Figura 7, a), b) y c) se puede observar que todas las temperaturas promedio de los elementos de la chimenea solar presentan un punto de inflexión a las 13:00 h, debido a la baja irradiación solar registrada (186 $\left.\frac{W}{m^{2}}\right)$.

TLATELPA-BECERRO, Ángel, RICO-MARTÍNEZ, Ramiro, URQUIZA-BELTRÁN, Gustavo y REYNOSO-JARDÓN, Elva Lilia. Análisis teórico de una chimenea solar con tres canales de flujo de aire. Revista de Operaciones Tecnológicas. 2019 


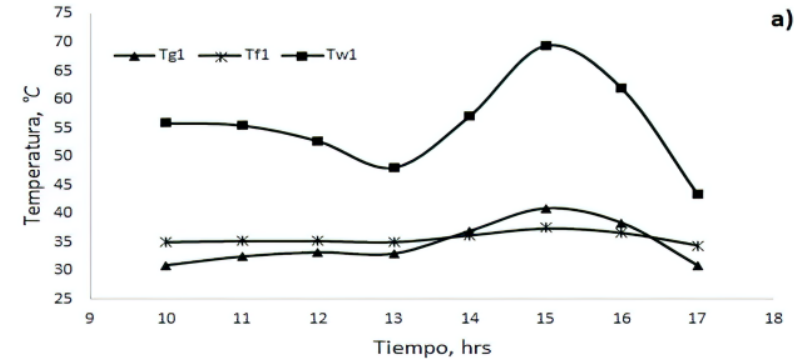

b)
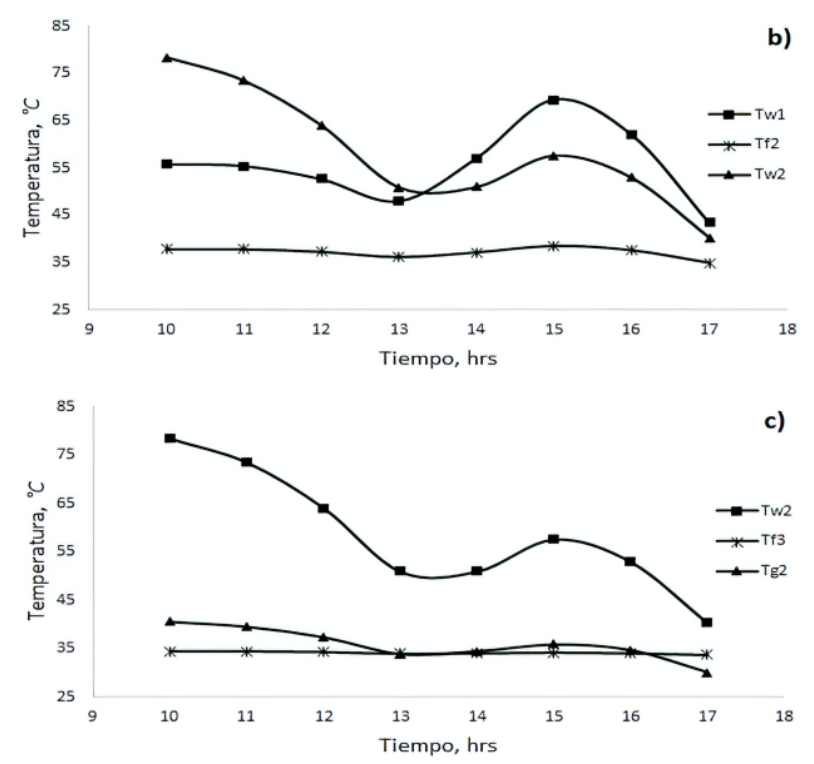

Figura 7 Temperaturas promedio vs tiempo. a) canal 1, b) canal 2 y c) canal 3. Se utiliza la relación de área de $\mathrm{A}_{0} /$ $\mathrm{A}_{\mathrm{i}}=1.0 \mathrm{y}$ el coeficiente de descarga de $\mathrm{Cd}=0.52$

Fuente: Elaboración Propia

La eficiencia térmica promedio y el caudal másico que sale de la chimenea solar en función de la radiación solar incidente se presenta en la Figura 8. Como se puede ver, el caudal másico alcanza su valor máximo a las 10:00 y a las 15:00 horas. Mismo comportamiento que el previsto para la eficiencia térmica.

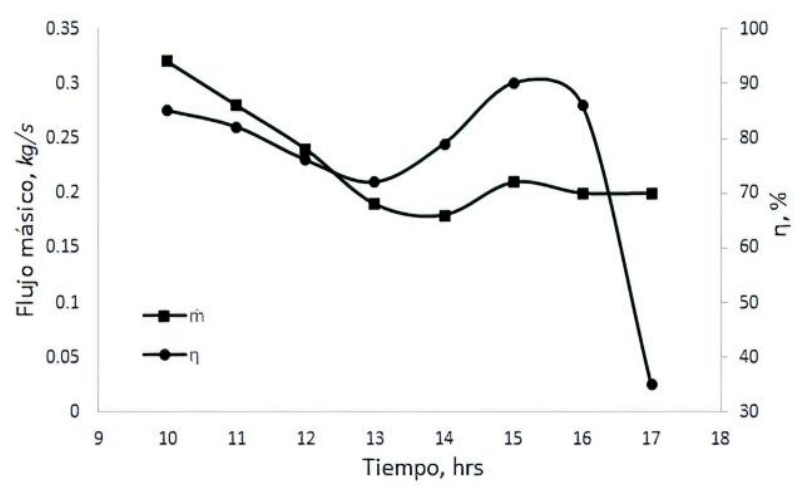

Figura 8 Caudal másico promedio $(\dot{m})$ y eficiencia térmica $(\eta)$. Se utilizaron la tasa de área de $\mathrm{A}_{0} /{ }_{\mathrm{Ai}}=1.0 \mathrm{y}$ el coeficiente de descarga de $\mathrm{Cd}=0.52$

Fuente: Elaboración Propia

\section{Conclusiones}

Se ha desarrollado un modelo matemático de una chimenea solar con tres canales de flujo de aire unidimensionales. Los resultados mostraron que las temperaturas de las placas metálicas $\left(T_{w 1}, T_{w 2}\right)$, las temperaturas del fluido del air $\left(T_{f 1}, T_{f 2}, T_{f 3}\right)$,y las temperaturas de las cubiertas acrílicas $\left(T_{g 1}, T_{g 2}\right)$ incrementan con la radiación solar.

El flujo máximo promedio y la eficiencia térmica se predijeron en $0.23 \frac{\mathrm{kg}}{\mathrm{s}}$ y $75 \%$, respectivamente, considerando la variación de la irradiación solar durante el día. Esta configuración exhibe un rendimiento mucho mejor que las chimeneas de un solo canal.

La configuración parece ser una alternativa viable para el diseño de sistemas de ventilación natural para reducir el consumo de energía convencional en edificios y puede aplicarse también a edificios industriales.

Para futuras investigaciones, es recomendable realizar un estudio paramétrico realizando cambios de dimensiones en la longitud, profundidad y entre el canal de hueco de la chimenea, para obtener los rendimientos de cada configuración, para propuestas de construcción experimental. Así mismo, realizar una simulación numérica en 3D con el uso de software ANSYS Fluent de la chimenea solar con acoplamiento a una casa habitación de características reales.

\section{Referencias}

Awbi H.B. (2003). Ventilation of building. London: Spon press.

Bansal N.K., Mathur R., and Bhandari M.S. (1993). Solar chimney for enhanced stack ventilation. Building and environment, 28, 373377.

Ong K.S. (2003). A mathematical model of a solar chimney. Renewable energy, 28, 10471060 .

Hirunlabh J., Kongduang W., Namprakai P., and Khedari J. (1999). Study of natural ventilation of houses by a metallic solar wall under tropical climate. Renewable energy, 18, 109-119. 
Lee, K.H. and Strand, R.K. (2009). Enhancement of natural ventilation in buildings using a thermal chimney. Solar energy, 41, 615621.

Imran A. A., Jalil J. M., and Ahmed S. T. (2015). Induced flow for ventilation and cooling by a solar chimney. Renewable energy, 78, 236-244.

Ghalamchi M., Kasaeian A., and Ghalamchi M. (2015). Experimental study of geometrical and climate effects on the performance of a small solar chimney. Renewable and sustainable energy reviews, 43, 425-431.

Sandeep K. P., Deepak P., and Ahmed M. R. (2014). Computational studies on the effect of geometric parameters on the performance of a solar chimney power plant. Energy conversion and management, 77, 424-431.

Gholamalizadeh E., and Kim M. H. (2016). CFD (computational fluid dynamics) analysis of a solar-chimney power plant with inclined collector roof. Energy, 107, 661-667.

Zhongting H., Bingqing L., Wei H., Dengyun H., Jie J., and Jinwei M. (2018). Performance study of a dual-function roof solar collector for chinese traditional buildings application. Applied thermal engineering, 128, 179-188.

Saifi N., Settou N., Dokkara B., Negrou B., and Chennouf N. (2012). Experimental study and simulation of airflow in solar chimneys. Energy procedia, 18, 1289-1298.

Karima E. A., and Saif W. M. (2012). Experimental and numerical studies of solar chimney for natural ventilation in iraq. Energy and buildings, 47, 450-457.

Yunus A.C. and Michael A.B. (2015). Thermodynamics. Mexico: McGrawHill/Interamericana Editores S.A. de C.V.

Duffie J.A., and Beckman, W.A. (1991). Solar engineering of thermal processes. New Jersey: John Willey \& Sons.

Incropera F.P., and De WITT D. P. (2002). Fundamentals of Heat and Mass Transfer. U.S.A: John Willey \& Son.
Bansal N.K., Mathur J., Mathur S., and Jain M. (2005). Modeling of window-sized solar chimneys for ventilation. Building and Environment, 40 (10), 1302-1308.

Modest M.F. (2003). Radiative Heat Transfer. New York: McGraw-Hill.

Mills A. F. (1999). Heat Transfer. U.S.A: Prentice Hall.
TLATELPA-BECERRO, Ángel, RICO-MARTÍNEZ, Ramiro, URQUIZA-BELTRÁN, Gustavo y REYNOSO-JARDÓN, Elva Lilia. Análisis teórico de una chimenea solar con tres canales de flujo de aire. Revista de Operaciones Tecnológicas. 2019 